\title{
Diagnóstico del estado de la Comunicación Promocional del Hotel Saratoga
}

\section{Diagnosis of the status of the Promotional Communication of the Saratoga Hotel.}

Arlette Arceo Martínez. ${ }^{1} \&$ Adriel Malvarez Cuello. ${ }^{2}$

\begin{abstract}
This research has the objective to diagnose the state of Promotional Communication in Saratoga Hotel, because of the importance for the hotel image. In the development we applied some techniques and methods to make the diagnosis of the communication in the hotel; analyzing the strengths and weakness of the facilities, as well as the opportunities and the threats that the environment brings to the hotel when talking about Promotional Communication. As results we achieved some important conclusions that distinguished the current situation of the hotel and they will be relevant in order to make new strategies in the future to improve its image and increase its sells.
\end{abstract}

Key Words: Communication, Promotional Communication, Saratoga Hotel.

\section{Resumen}

La presente investigación se realizó con el objetivo de diagnosticar el estado de la Comunicación Promocional en el hotel Saratoga, por la importancia que esta representa para la imagen que se proyecta del hotel. Para ello se realizó un estudio basado en los fundamentos teóricos que respaldan el tema en cuestión. En el desarrollo del trabajo se aplicaron las técnicas y métodos correspondientes para realizar el diagnóstico pertinente de la comunicación en el hotel; analizando las fortalezas y debilidades que presenta la

\footnotetext{
${ }^{1}$ Facultad de Turismo-Universidad de La Habana, Cuba arceomartinezarlette@gmail.com

${ }^{2}$ Facultad de Turismo-Universidad de La Habana, Cuba. adrielmalva2017@gmail.com
} 
instalación, así como las oportunidades y amenazas que le brinda el entorno a la entidad en materia de Comunicación promocional. Como resultado se obtuvieron varias conclusiones importantes que caracterizan la situación actual del hotel, que serán relevantes para trazar nuevas estrategias futuras en aras de mejorar la imagen del hotel y aumentar sus ventas.

Palabras Claves: Comunicación, Comunicación Promocional, Hotel Saratoga

\section{Introducción}

Actualmente, el sector turístico está atravesando por un marcado grado de complejidad debido a la globalización de la oferta y al conocimiento cada vez mayor de la demanda, lo que ha provocado que los destinos turísticos tengan que trazarse nuevas estrategias. En el caso de la hotelería al tener como base de su desarrollo turístico el mismo producto: el alojamiento, ha provocado que estos tengan que diferenciarse de la competencia y posicionarse dentro de sus mercados de una manera más efectiva. Esta situación hace que el directivo se concentre en la búsqueda de nuevas alternativas que les permitan ser más competitivos o recurrir a nuevas fórmulas o estrategias de marketing y comunicación para poder responder a las exigencias de la demanda. Es importante añadir que dentro de estas estrategias se encuentra la Comunicación Promocional, la cual aplicada de manera correcta puede contribuir al fortalecimiento de la imagen del hotel en el mercado meta, así como lograr una diferenciación con respecto a la competencia, provocando un mayor incremento en las ventas y en el arribo de visitantes. (Perelló, 2010)

La Comunicación Promocional en el ámbito del turismo adquiere una importancia relevante y según (Ezquerra y Martínez, 2007), sus objetivos, enfocados hacia el turismo podrían analizarse bajo la perspectiva de: "captar la atención de aquellas personas para quienes vacacionar es una forma tradicional de recreación; incrementar la dimensión del mercado turístico, atrayendo hacia éste personas que no suelen viajar con propósitos turísticos; contrarrestar el flujo de turistas durante las llamadas épocas bajas, convirtiendo a éstos en destinos turísticos atractivos fuera de las épocas tradicionales".

La comunicación promocional resulta necesaria para "comunicar la existencia del producto/servicio, dar a conocer sus características, ventajas, necesidades que satisface... persuadir al comprador potencial de los beneficios que reporta el producto/servicio ofrecido y en definitiva tratar de estimular la demanda, recordando la existencia del producto/servicio y sus ventajas, a fin de evitar que los usuarios reales del producto/servicio sean tentados por la competencia y adquieran otras marcas" (Rivero, 2005).

Es por ello que puede ser catalogada entonces, como una intención comunicativa en esencia, que luego se gestiona a partir de las herramientas que integran el mix de 
comunicación. Se utiliza para comunicarse tanto con el público en general como con cualquiera de los eslabones de la cadena distributiva. Puede incluir, por tanto, varios soportes: impresos, audiovisuales o digitales, en dependencia de los objetivos trazados para cada una de las herramientas integrantes del mix. De esta forma cuando se habla de promoción se integran acciones tanto de Relaciones Públicas (ferias y presentaciones) como de Publicidad (Ezquerra y Martínez, 2007).

Por lo que partiendo de la importancia que tiene La Comunicación Promocional para cualquier instalación el presente trabajo se encuentra enfocado en lograr el siguiente objetivo general:

Objetivo General: Diagnosticar el estado de la Comunicación Promocional del hotel Saratoga

\section{Objetivos específicos}

1. Determinar los fundamentos teóricos y metodológicos que sustentan la investigación.

2. Caracterizar la Empresa Mixta Hotel Saratoga S.A., en materia de Comunicación promocional.

3. Identificar las debilidades, amenazas, fortalezas y oportunidades del Hotel Saratoga en materia de Comunicación Promocional.

\section{Tareas por objetivo}

1. Determinación de los referentes teóricos metodológicos que sustentan la investigación.

2. Caracterización de la Empresa Mixta Hotel Saratoga S.A.

3. Identificación de las debilidades, amenazas, fortalezas y oportunidades del Hotel Saratoga S.A. en materia de Comunicación Promocional.

\section{Metodología.}

Para la ejecución del presente trabajo se emplearon diferentes métodos de investigación mediante técnicas cuantitativas y cualitativas. (Sampieri, 2010) En correspondencia a los métodos teóricos se utilizaron el análisis y la síntesis lo cual permitió identificar los elementos que condicionan la situación problémica, así como establecer la relación entre ellos; la inducción y deducción a los fines de profundizar en el conocimiento de lo general a lo particular. También se llevó a cabo el método histórico-lógico para la revisión bibliográfica y documental, en vistas al establecimiento del marco teórico referencial y las características del tema en cuestión.

Se aplicó el método empírico a través de técnicas como la encuesta y la entrevista con el fin de definir aspectos fundamentales de la investigación (conocimiento de los clientes 
sobre la Comunicación Promocional del hotel y el impacto que tiene en el mercado), y además se empleó la observación, para ver los distintos procesos llevados a cabo por la instalación en materia de promoción. Otra técnica utilizada fue el análisis DAFO, En el caso de esta sirvió para determinar las debilidades, amenazas, fortalezas y oportunidades de la empresa con relación a la Comunicación Promocional.

También fue empleado el método estadístico a través de diferentes técnicas como: el muestreo que permitió seleccionar una muestra representativa con porcentajes elevados de niveles de confianza dentro de la población a investigar, ello con el objetivo de aplicar las encuestas e interpretar sus resultados a través de la técnica de análisis de frecuencia, para validar los resultados de las encuestas.

\section{Resultados.}

\section{Caracterización del Hotel Saratoga}

La Empresa Mixta Hotel Saratoga S.A. fue constituida al amparo de la Ley No.77, Ley de la Inversión extranjera, por un período de tiempo de 25 años mediante la Escritura Notarial No.1221 de fecha 4 de julio de 1996 y el Hotel Saratoga fue inaugurado posteriormente en el año 2005. Inicialmente, se constituyó una empresa mixta entre Saratoga S.A. (Inversor británico) y Habguanex S.A. que se encontraba subordinada a la Oficina del Historiador de la Ciudad. Posteriormente se produjo la adquisición de Habaguanex por el grupo de Administración empresarial en el año 2012, por lo que el hotel pasó a formar parte de la cadena Gaviota S.A. (Hotel-Saratoga.com, s.f.)

El Objeto Social de la empresa es la adaptación, reconstrucción, rehabilitación, remodelación, restauración y posterior explotación del Hotel Saratoga, presidiendo una ubicación de privilegio de La Habana Vieja, la esquina del Paseo del Prado y Dragones. Su misión es: «Brindar servicios de alojamiento y comercializar un producto hotelero único en un entorno de genuino valor arquitectónico, histórico-cultural, diseñado para un cliente exigente».

Su visión es: «Somos un producto hotelero único ubicado en el entorno sociocultural y patrimonial de La Habana, elegido por su tranquilidad, confort y exclusividad, con un equipo estable que se distingue por su profesionalidad, alcanzando los resultados económicos financieros esperados, en convivencia armónica con la comunidad».

El hotel presenta un sistema de valores bien definidos con su forma, período y responsable de medición, los cuales se relacionan en la siguiente tabla: 


\section{EDDigital}

ISSN: 2661-6831

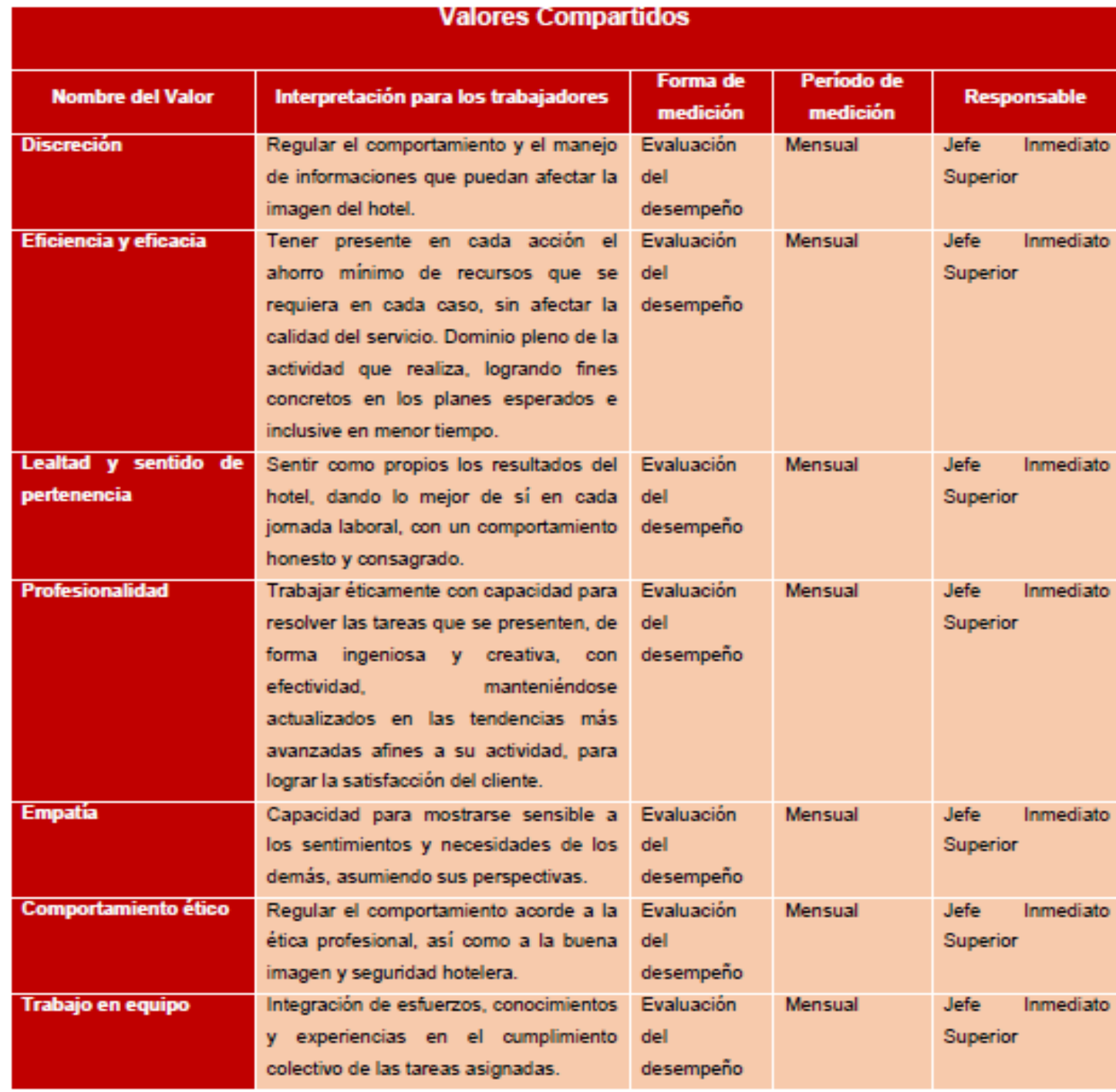

Tabla1. Valores Compartidos.

Fuente: Recursos Humanos Hotel Saratoga.

\section{Identidad Visual del hotel}

El hotel cuenta con un manual de identidad visual perteneciente a Saratoga S.A., en el que se pautan los elementos gráficos y sus normas de aplicación, con el fin de servir de herramienta eficaz para controlar todos aquellos aspectos que garantizan la coherencia en la imagen de su identidad. Los autores solo tuvieron acceso a determinados capítulos del manual, pues en su gran mayoría este es confidencial y, a continuación, se explican las características más generales de la identidad visual del hotel.

\section{Signos básicos de identidad}

En el manual los signos básicos de identidad están referidos a la tipografía, estructura y color de la marca, así como las diferentes variantes de color a utilizar. Estos signos de 
identidad no son solo para el logotipo de la entidad, sino también para los diferentes identificadores de los distintos lugares del hotel, tales como los bares y los restaurantes.

\section{Gama cromática}

El color corporativo es el Pantone 7427 C y este se aplicará en la marca y en todos los elementos internos y externos relacionados con el hotel. Además, se establecen las variantes de color para el uso del color corporativo: Color positivo y negativo (Pantone $7427 \mathrm{C}$ y blanco), negro positivo y negativo y se recomienda el uso del Pantone $871 \mathrm{c}$ (dorado) como color complementario para aplicaciones especiales donde se requiera transmitir distinción y elegancia.

\section{Slogan}

No se apreció la existencia de un slogan en el manual de identidad visual del hotel.

\section{Aplicaciones en diferentes soportes}

Los signos básicos de identidad del hotel tienen varias aplicaciones:

Poseen aplicaciones en toda la papelería de la entidad: papel de carta ejecutivo, papel de fax, sobre de cartas, sobre de documentos, tarjeta de presentación, tarjeta ejecutiva, parque de vehículos, tarjeta de cortesía, tarjeta de bienvenida, tarjeta para mensajes, porta tarjeta de bienvenida, porta tarjeta para llave magnética, listado de servicio de lavandería, directorio telefónico, block para directorio telefónico, colgante para desayuno, colgante no moleste, ticket para equipaje, tarjeta para huésped, sobre para tarjeta de huésped, carpeta para el huésped, block de notas, carta de almohadas, etc. Además, los colores se pueden apreciar en los uniformes de todas las áreas del hotel.

Además, tiene aplicaciones para la promoción pues regula cómo serán los anuncios en las revistas, las jabas promocionales de papel, pullover, etc.

\section{Técnicas de Comunicación Promocional que se aplican en el hotel.}

En el hotel se aplican las distintas técnicas de Comunicación Promocional existentes:

- Publicidad: en el hotel existe un video promocional, que aunque no está en ningún medio de comunicación, si se reproduce en las pantallas que se encuentran distribuidas en la zona de la entrada y el lobby del hotel, así como en el restaurante Anacaona. Además, existe un mensaje publicitario para la radio que se puede escuchar en la emisora Radio Taíno, para promocionar las nuevas ofertas del Restaurante. En la fachada del hotel hay un cartel en el que se está promocionando el festival gastronómico que se está realizando, en honor al 499 aniversario de la Villa de San Cristóbal de La Habana y también se reparten sueltos promocionales, 
así llaveros, abanicos, bolsas, pisapapeles, etc., todos con el logotipo de Saratoga. Es importante mencionar también los anuncios publicitarios en las diferentes revistas especializadas.

- Relaciones Públicas: Como parte de las relaciones públicas, el hotel se preocupa por la comunidad en la que se encuentra enclavado, por lo que mantiene un convenio con la Escuela Primaria Concepción Arenal (que se encuentra ubicada al lado del hotel), manteniendo una relación de mutuo beneficio. Sin embargo, como aspecto negativo, cabe destacar que el hotel no posee un programa de fidelización a pesar de la elevada cantidad de clientes repitentes que presenta el mismo. Además, la plaza de relacionista público está combinada con la de especialista de calidad, realizando estas funciones la misma persona, lo cual puede entorpecer el trabajo de ambos cargos por separado. romoción de ventas: Como forma de promocionar las ventas en el hotel son realizadas las siguientes acciones: es utilizado el mailing dirigido hacia los clientes del establecimiento con las nuevas ofertas que presenta la instalación; se realizan en el hotel decoraciones acorde con la festividad a realizar (navidad, año nuevo, día de los enamorados, etc) y promoción de las ofertas especiales de las mismas, expuestas en las pantallas del establecimiento. Además, por vía telefónica y por medio de la recepción, son recordadas a los clientes las fechas de los días festivos, en aras de que participen en las actividades que realiza el hotel

- Fuerza de ventas: La fuerza de venta fue solamente apreciada en el buró de ventas perteneciente a la Agencia de Viajes Gaviota y en algunas ocasiones en la Recepción del hotel donde se observó como en algunos momentos el personal hacía hincapié en la venta de un producto determinado del mismo.

\section{Aplicación de los lineamientos de la política del turismo en el hotel.}

El hotel aplica varios de los lineamientos pertenecientes a la política de turismo. (Ministerio del Turismo) Entre ellos el 255, pues el principal objetivo del hotel como entidad turística, consiste en la captación directa de divisas, y en aras de maximizar el ingreso medio por turista el hotel crea alternativas para que los clientes consuma la mayor cantidad de productos tanto dentro como fuera de la entidad; por otra parte se le da cumplimiento al lineamiento No 258, pues el directivo del hotel trata de aprovechar por todos los medios posibles vender los productos-servicios que ofrece la instalación y por último el lineamiento No 260 mediante la creación de nuevas ofertas que hagan el hotel más atractivo para los clientes.

Particularmente con respecto al lineamiento No 261, se pretende a corto plazo, diversificar la oferta del hotel utilizando como vía de promoción para ello, diferentes medios de comunicación, principalmente a través de las redes sociales por el impacto que han generado globalmente. 
Por último, también se le da cumplimiento al lineamiento No211 que plantea:

"Perfeccionar las formas de comercialización, utilizando las tecnologías más avanzadas de la información y la comunicación y potenciando la Comunicación Promocional”.

Este lineamiento se ve reflejado en todas las acciones que realiza el hotel para promoción de sus productos, principalmente con el uso de las TIS destacándose las cuentas en las diferentes redes sociales. \}

\section{Uso y aplicación de las Tecnologías de Información y Comunicación para la promoción del hotel.}

Gran parte de la promoción del hotel está dada por el uso de las Tecnologías de la Información y la Comunicación. Esto se ve evidenciado en que la entidad posee una página web en la que se publica el booking actualizado del hotel y mediante la cual los clientes pueden reservar y pagar online. Además, existen cuentas en redes sociales como: Facebook, Twitter, Instagram, Pinterest y Google Plus.

El hotel tiene un contrato con la Agencia Española Bidaiondo, que es la empresa encargada de mantener actualizada la página web y de ofrecer el servicio de la plataforma de pago virtual. Dicha agencia es también quien realiza las publicaciones y maneja las cuentas en las redes sociales, para lo cual, el departamento comercial del hotel debe enviarle las fotos y los textos a publicar. Además, las redes sociales del hotel están enlazadas a las cuentas privadas de personal estratégico del hotel, tales como: el director general, el informático, el comercial y la especialista de calidad y relaciones públicas. Esto se hace con el objetivo de que ellos puedan subsanar inmediatamente cualquier error en la información publicada, obtener más seguidores e, incluso, realizar publicaciones; aunque este último no suele realizarse.

\section{Técnicas aplicadas}

\section{Resultados de la Guía de Observación}

La observación tuvo un carácter directo no participante, y se realizó con el objetivo de apreciar elementos de trascendencia para la investigación, en aras de arribar a conclusiones.

Como resultado de aplicar la Técnica de Observación se obtuvo la siguiente información: La instalación se encuentra bien identificada por su nombre, logo y cadena a la cual pertenece, los que se encuentran en lugares estratégicos (la entrada del Hotel, en el lobby reflejado en las pantallas, y en los soportes de comunicación que utiliza la empresa: Files, carpetas, documentos oficiales, etc).

El hotel presenta un Manual de Identidad Corporativa, en el cual se ven reflejadas las normas que se deben seguir para imprimir la marca y el logotipo en los diferentes soportes 
internos y externos de la compañía, con especial hincapié en aquellos que se mostrarán al público, en él se incluyen los colores y las tipografías corporativas. (Saratoga) Dicho manual se ve reflejado en los distintos materiales que posee la entidad, en los uniformes de los empleados, en la ambientación y decoración del lugar, en la señalética que muestra la instalación. Todo lo anterior se encuentra en coherencia con la imagen que quieren mostrar el hotel.

En cuanto a la ambientación como la decoración de las diferentes áreas se pudo observar que estas brindan gran confort, elegancia y lujo, donde se combinan hermosos elementos decorativos de principios de siglo con espacios y detalles modernos, matizados por un toque de ingenio y buen gusto. Las inmediaciones del hotel son, hay que reconocerlo, un poco ruidosas y no muy agradables desde el punto de vista estético. Sin embargo, esta pequeña deficiencia está más que compensada por la ubicación privilegiada del lugar. Además, contiene un sofisticado y atrevido diseño de interiores que marca un nuevo tono para los hoteles del área, evocador del espíritu cosmopolita, singular en grandes ciudades como Nueva York y otras. El bar del entrepiso, situado bajo un tragaluz del techo, es el vivo ejemplo de esta sensación, con su mobiliario diverso, elevadas palmeras y un gran mural que muestra un paisaje cubano, se nombra "Bar Mezzanine". Igual de atractivos son; el restaurante Anacaona, el bar adyacente (con el mismo nombre) y el restaurante de la azotea "Mirador Saratoga", este ofrece hermosas vistas del Paseo del Prado y de otros lugares de interés como la Fábrica de Tabacos Partagás.

Las áreas se encuentran bien identificadas, tanto por su decoración como por la señalética que nos brinda el lugar. Por lo general resultan lugares espaciosos y bien equipados según las características del mismo.

Con respecto al personal se observó cómo el mismo se encuentra correctamente uniformado en correspondencia con la función que realiza en el hotel (recepcionistas, botones, guardias de seguridad, dependientes gastronómicos, etc). Todos los uniformes poseen una identificación con el nombre de la persona que lo porta. Y además poseen una adecuada presencia y cordialidad en el recibimiento de los clientes.

El personal de forma general presenta una buena comunicación interpersonal reflejada en la cordialidad a la hora de comunicarse con los clientes tanto internos como externos, y además en la atención brindada y la forma de expresarse. Proyectando una imagen de profesionalidad y preparación hacia os clientes. Por otra parte, en cuanto a la relación de los trabajadores con el directivo se ve reflejado el respecto y la buena comunicación entre ellos, manifestado en el constante flujo de información existente en la entidad, que mantiene actualizado al personal, siendo estos partícipes de las acciones a tomar por la empresa, pues existe un buen equipo de trabajo donde todos colaboran para lograr un objetivo en común: vender el hotel como producto. 
El hotel presenta, aunque escasos, soportes de comunicación. Ejemplo de los que son visibles son los siguientes: las pantallas en el lobby con el nombre de la instalación y la cadena hotelera a la cual pertenece; las principales ofertas de la instalación y las redes sociales en las cuales están presentes; algunos posters con imágenes de la instalación, y el nombre del hotel a la entrada en un tamaño significativo, además existe un punto de venta de la Agencia de Viajes Gaviota, donde se muestran varios de los servicios que ofrece la entidad en cuestión.

Aunque en el hotel no exista gran variedad de soportes de comunicación, si se le atribuye importancia a las técnicas de comunicación promocional con el objetivo de llamar la atención, despertar el interés, conducir el deseo y llevar a la acción de comprar el producto. Ejemplo de ello se ve reflejado en: en la carta menú del restaurante donde se apreciaron técnicas de merchandising como la técnica de adjetivación (que facilitan identificar y recordar nombres de ofertas atractivas) y la técnica de fijación de precios (que propicia obtener ingresos extras con discretos aumentos o rebajas de precios con mínima dificultad). La promoción de ventas se vio reflejada en el buró de venta perteneciente a la Agencia de Viajes Gaviota.

\section{Entrevista a la Directora Comercial}

La entrevista se realizó con el objetivo de conocer los criterios y enfoques que determinado personal directivo del hotel objeto de estudio, tienen respecto a la Comunicación Promocional, su desempeño en el hotel y la manera en que debía enfocarse la gestión de la misma.

Los resultados de esta Entrevista, arrojaron la siguiente información:

La especialista manifiesta que la comunicación es un aspecto clave para comercializar el hotel, no solo mediante la promoción del alojamiento y las ofertas gastronómicas del mismo, sino también promocionarlo como destino. Pues la información que brinde e hotel de sus servicios, así como la imagen que muestre del mismo, será un factor determinante para que el cliente lo escoja o no para pasar su estadía, pues, aunque tengas un producto muy bueno, si el mundo no lo conoce, no se vende.

La entrevistada no mostró tener conocimiento sobre la existencia de un manual de Identidad Corporativa, por lo que se puede decir que a pesar de que exista una coherencia entre lo que pauta 1 manual y las normas que se establecen en la empresa, parte del personal no tiene conocimiento de la existencia de documentos por los que se rige la entidad.

Por otra parte, mencionó que las principales acciones de Comunicación Promocional que emprende el hotel son las siguientes: a través de las redes sociales (Facebook, Twitter, Pinterest, Instagram y Google plus), participación en ferias (FITUR, FIAR), radio, revista 
cultural (Excelencia), sueltos, correos electrónicos, el boca a boca. Sin embargo en ocasiones estas realmente no son efectivas pues muchas veces no se encuentran enfocadas al público objetivo adecuado. Además, plantea que para mejorar el estado de la Comunicación resulta necesario aprovechar todos los elementos que se tengan a la mano y las oportunidades que brinde tanto el micro como el macro entorno.

Con respecto a la destinación de presupuesto para realizar actividades de promoción comenta que la entidad no tiene autonomía para decidir $100 \%$ sobre todos los proyectos $\mathrm{y}$ actividades que quisiera hacer, y no resulta de igual modo para la entidad una prioridad, pues se enfocan en resolver otros problemas que tienen para la empresa mayor importancia.

Por otra parte, alega que las principales fortalezas que presenta el hotel en materia de promoción lo constituye el prestigio ganado por el hotel durante todos estos años, además de la preparación del personal y el trabajo en equipo, además del elevado sentido de pertenencia que tienen los trabajadores por la instalación. En cuanto a las debilidades se encuentra: que muchas veces no cuentan con la aprobación para la realización de actividades de promoción, además de no contar con un presupuesto destinado para ello. Por último, hace alusión a que el hotel no cuenta con personal suficiente para encargarse de las acciones de comunicación, sin embargo, el que se encuentra es eficiente y se encuentra capacitado para hacer el trabajo.

\section{Entrevista al Director General}

Como resultado de la entrevista realizada al Director General del hotel, Miguel Perdomo García, se obtuvo lo siguiente:

El Director General del hotel considera que la Comunicación Promocional son todas aquellas técnicas o herramientas de la comunicación que pueden y son utilizadas para promocionar un producto o servicio. Expresó que esta es de vital importancia para el Hotel Saratoga, puesto que constituye una herramienta fundamental para hacerle llegar sus productos a los clientes. Sin embargo, en los últimos años, dicha herramienta, no está siendo lo suficientemente efectiva, pues este ha sido uno de los factores que ha estado incidiendo en la baja ocupación que presenta el hotel.

El director señaló la existencia de las políticas y el manual de identidad para manejar la comunicación y explicó que eran estrictamente confidenciales, pues, por una parte, todo el tema de la identidad era manejado por el grupo inversor y las políticas a seguir eran dictadas por la cadena Gaviota S.A. También explicó que, aunque toda la comercialización era llevada a cabo por el director extranjero, en representación del inversor, el hotel realizaba todas las acciones posibles de comunicación promocional para intentar mejorar la situación de ocupación existente. Algunas de las acciones promocionales que realizan son: mensajes publicitarios de las ofertas del hotel en la radio, 
brochures, publicaciones en las redes sociales y en la pagina web, anuncios en revistas especializadas tales como Hosteltur y Excelencias, así como objetos promocionales: abanicos, llaveros, pisapapeles, agendas, lápices, bolsas, tasas, etc.

Él considera que estas acciones son efectivas, pero no lo suficiente, como para atraer a grandes volúmenes de clientes, por lo que en la actualidad se está realizando un estudio de mercado en el hotel para evaluar las necesidades de su segmento de mercado y, de esta manera, redefinir su estrategia en cuanto a comunicación promocional. En cuanto al presupuesto, el director manifestó que en este momento el presupuesto que se destinaba a esta herramienta no era muy elevado y que debían ser muy cuidadosos en las decisiones que se tomaran debido a los bajos ingresos que está presentando el hotel por el descenso de la ocupación. Además, las decisiones en este sentido no dependían de él, sino de la cadena Gaviota S.A. En su opinión, el personal del hotel que realiza las labores de comunicación no es suficiente pues, por ejemplo, en el caso de la relacionista pública, esta es la misma persona que realiza el trabajo de especialista de calidad y de community manager; aunque esto no evita la eficiencia del trabajo de la misma.

\section{Encuesta a los trabajadores}

Durante la investigación se aplicó un total de 40 encuestas a la muestra seleccionada del hotel de un total de 168 trabajadores. Del total de encuestas aplicadas, fue necesario desechar 5, a consideración de los autores, debido a que estaban incompletas y entorpecerían la obtención de datos fehacientes. A continuación, se presentan los resultados obtenidos a partir del análisis de las mismas:

Primeramente, se puede apreciar que la totalidad de los encuestados, consideran importante la adquisición de conocimientos sobre Comunicación, Comunicación Promocional e Interpersonal para los trabajadores del Turismo, lo cual se considera un aspecto positivo para el perfeccionamiento laboral teniendo en cuenta la auténtica relevancia de la disciplina para el desarrollo del Turismo.

Todos coinciden en que las personas encargadas de la comunicación en el hotel es la especialista de Relaciones Públicas de conjunto con el departamento comercial, aunque resulta interesante como algunos afirman que los mencionados anteriormente son los principales, pero a la larga, todos los departamentos del hotel (Recepción, Ama de Llaves, Servicios Técnicos, entre otros), tributan a la Comunicación promocional del mismo.

Se pudo observar que, de manera general, todos los encuestados conocen las técnicas de Comunicación Promocional que se desarrollan en el hotel, siendo estas: la promoción en ferias de turismo, las cuentas en redes sociales, las publicaciones en la página web, etc. En la Figura No.1, que se muestra a continuación, se puede observar que el 13\% de los encuestados considera que los clientes tienen una Buena imagen de la instalación, 
mientras que el $87 \%$ piensa que tienen una imagen Muy buena; es decir que, para los trabajadores, el hotel proyecta una excelente imagen.

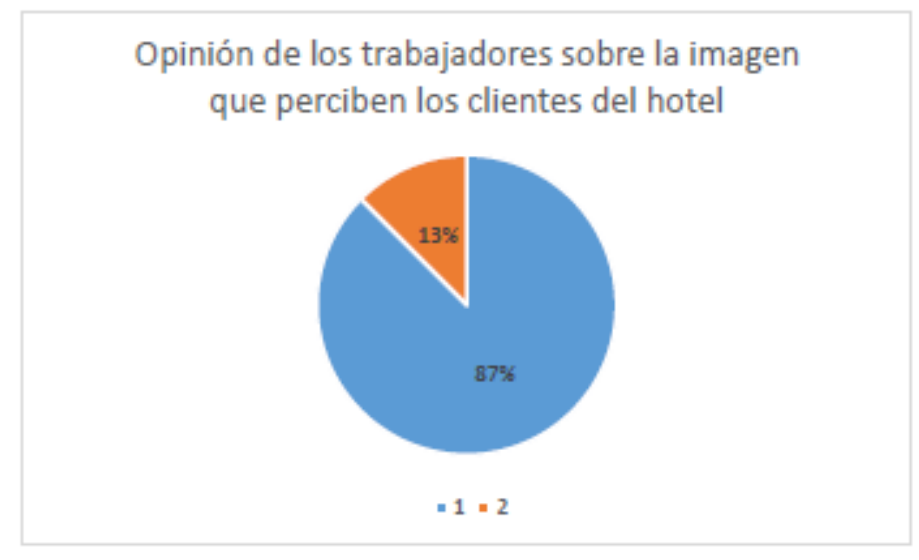

Figura 1. Opinión de los trabajadores sobre la imagen que perciben los clientes del hotel. Fuente: Elaboración propia.

El $100 \%$ de los encuestados expresó que su trabajo contribuye a la Comunicación Promocional del hotel, lo cual significa que los trabajadores están conscientes de la importancia que tiene su comportamiento y profesionalidad en su puesto de trabajo.

En la Figura No.2, se muestra que solo el $6.25 \%$ de los encuestados, contestó que no sabe si la comunicación promocional es efectiva o no, sin embargo, el $93.75 \%$ piensa que si lo es. Esto refleja que, para la mayoría de los trabajadores, el hotel está realizando un buen trabajo en este sentido.

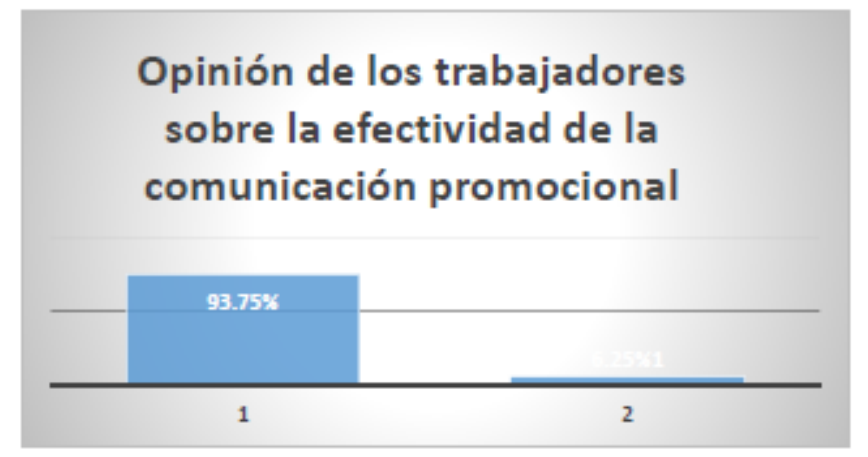

Figura 2. Opinión de los trabajadores sobre la efectividad de la comunicación personal Fuente: Elaboración propia.

La totalidad de los encuestados contestó que recibe la información de trabajo a través de murales, reuniones de información, correo electrónico y por vía telefónica. Esto evidencia la variedad de canales de información lo cual favorece la transmisión de la misma de forma interna.

Se puede observar en la Figura No.3 los pesquisados consideran que los estudiantes de Licenciatura en Turismo deben adquirir determinados tales como: Proceso de comunicación y elementos que lo integran (43.75\%), Tipos de comunicación promocional 
(68.75\%), Comunicación Interpersonal (75\%), Construcción de mensajes (37.5\%), Estructura y funcionamiento de las agencias publicitarias (6.25\%), Comunicación verbal y extraverbal (56.25\%), Técnicas o elementos de Comunicación Promocional (68.75\%), Relaciones Públicas (87.5\%), Marketing (93.75\%), Publicidad e imagen turística $(93.75 \%)$, Posicionamiento turístico (75\%), Planes de Comunicación Promocional $(31.25 \%)$.

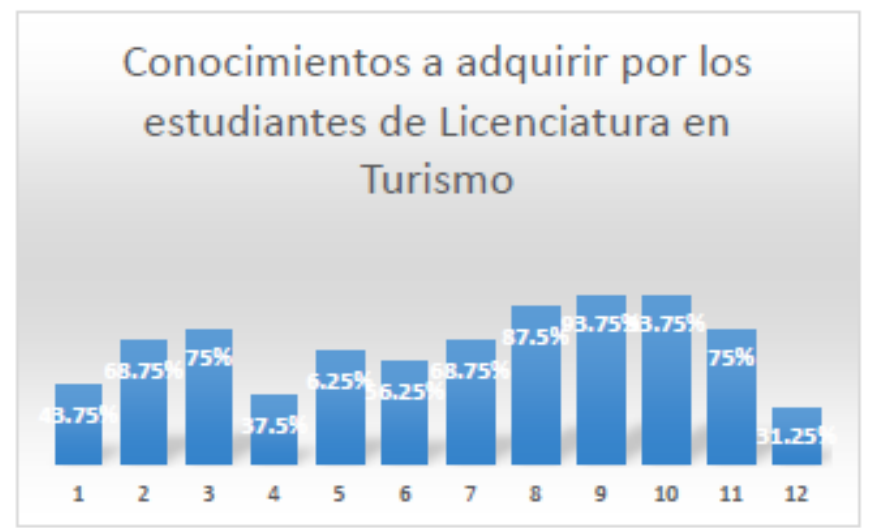

Figura3.Conocimientos a adquirir por los estudiantes de Licenciatura en turismo. Fuente: Elaboración propia.

Con estos resultados, se puede decir que los trabajadores están conscientes de la importancia que tiene la adquisición de conocimientos sobre Comunicación para un futuro Licenciado en Turismo. De hecho, los autores consideran que estos resultados se deben a que ellos mismos pueden carecer de algunos de estos conocimientos y los necesitan para su trabajo diario, lo cual se demuestra, primeramente, en la Figura No.4 que se muestra a continuación:

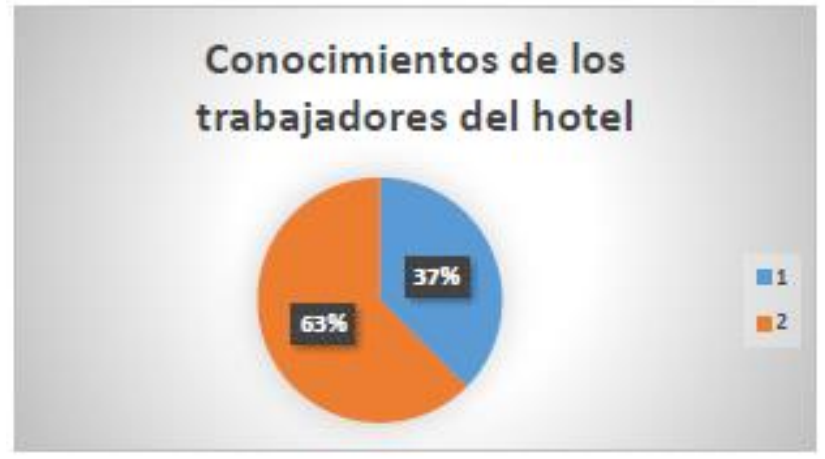

Figura 4. Conocimientos de los trabajadores del hotel. Fuente: Elaboración propia.

En la anterior figura se muestra que $37 \%$ de los encuestados asegura que los trabajadores del hotel poseen los conocimientos ya mencionados, mientras que el $63 \%$, piensa que no todos los poseen. Además, el $75 \%$ de los pesquisados afirma que les gustaría recibir capacitación en algunos de estos conocimientos, frente a un $6 \%$ que no está interesado, mientras que el 13\% dice no tener tiempo para ello (Obsérvese la Figura No.5). 


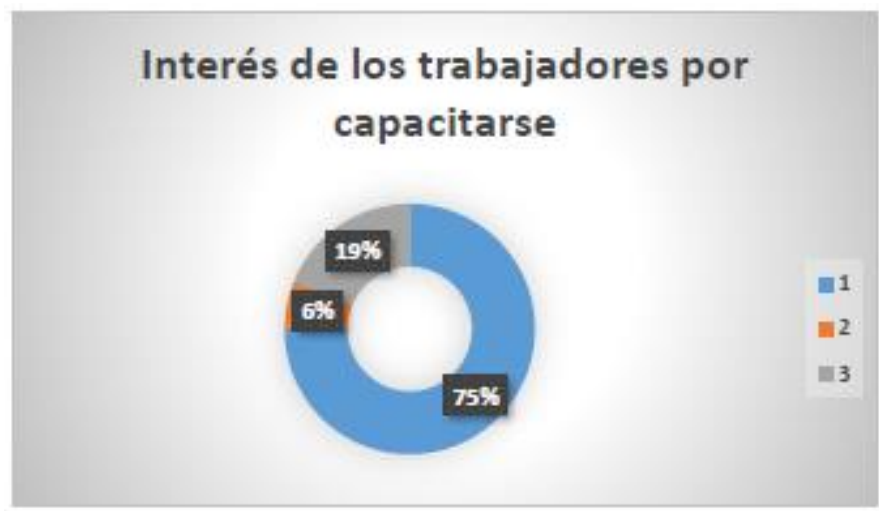

Figura 5. Interés de los trabajadores por capacitarse.

Fuente: Elaboración propia.

Cabe destacar, que los trabajadores que desean capacitarse están interesados fundamentalmente en el Marketing, el Proceso de Comunicación y los elementos que lo integran, la Publicidad e imagen turísticas, la comunicación verbal y extraverbal, así como las Técnicas o elementos de Comunicación Promocional.

\section{Encuesta a los clientes.}

Durante la investigación se aplicó un total de 16 encuestas (Ver Anexo No.5) a la muestra de clientes seleccionada. Del total de encuestas aplicadas, fue necesario desechar 3, a consideración de los autores, debido a que estaban incompletas y entorpecerían la obtención de datos fehacientes. A continuación, se presentan los resultados obtenidos a partir del análisis de las mismas:

El 17\% de los encuestados estaba visitando el hotel por primera vez, mientras que el $83 \%$ no, siendo esa la segunda o tercera vez que lo hacían (Véase la Figura No.6)

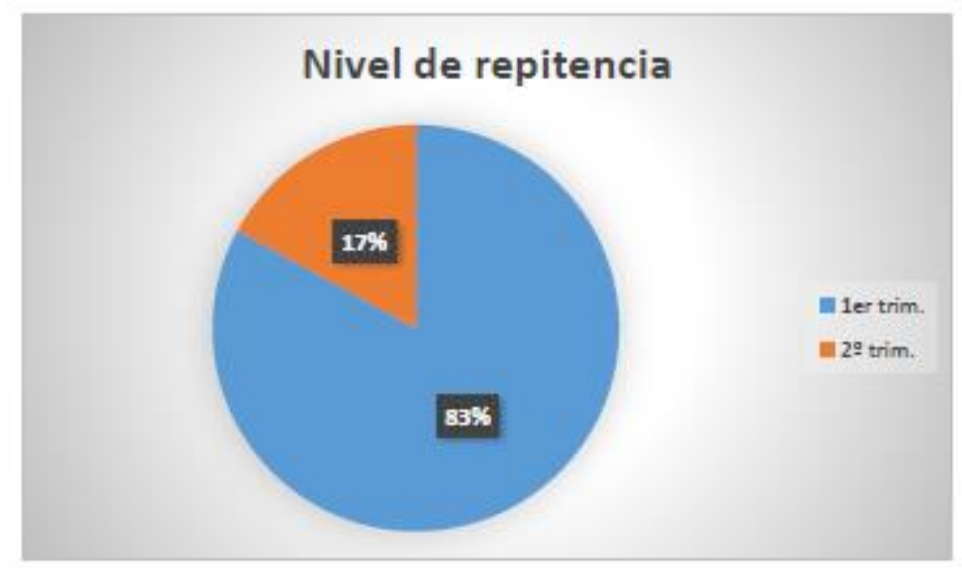

Figura 6. Nivel de repitencia.

Fuente: Elaboración propia.

Los clientes pesquisados conocieron de la existencia del Hotel Saratoga a través de una página web $(47.8 \%)$, a través de las redes sociales $(37.6 \%)$, a través de una agencia de 
viajes o turoperador (56.75\%), a través de una revista (29.25\%), por recomendación de un amigo o familiar (39.87\%). En su gran mayoría, decidieron hospedarse en el hotel por la fama que goza el mismo de haber hospedado en sus habitaciones a numerosas figuras de gran popularidad a nivel internacional y líderes de opinión.

La Figura No.7 muestra los principales atributos que perciben los clientes en la imagen del Hotel Saratoga: Confiabilidad (84\%), Tranquilidad (98\%), Lujo (96.25\%), Confort (88\%), Calidad (91\%), Experiencia (75\%). Esto significa que la imagen que perciben los clientes tiene unos atributos muy bien definidos, pues si bien, no todos los encuestados opinan lo mismo, si coinciden en su elección con por cientos bastante elevados.

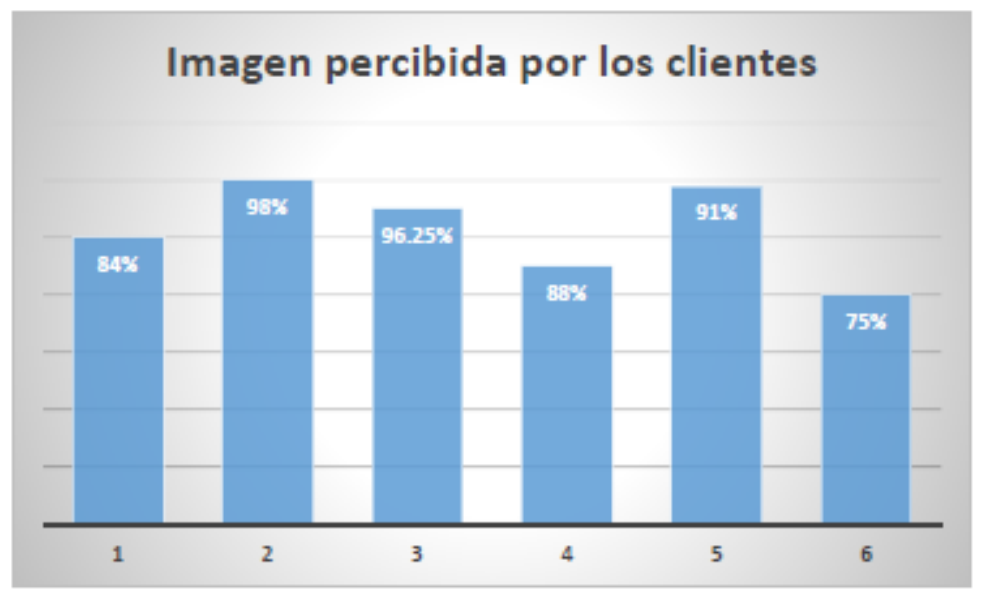

Figura 7. Imagen percibida por los clientes.

Fuente: Elaboración propia.

El $62 \%$ de los clientes conocen los colores que identifican al hotel, mientras que el $38 \%$ no los conoce. Por otra parte, el $77 \%$ de los encuestados conoce el logotipo del hotel frente a un $23 \%$ que no lo conoce. El $49 \%$ de los clientes valoró los servicios del hotel como excelentes y un $51 \%$ los calificó de buenos, siendo relevante que ninguno de los pesquisados los consideró regulares o malos.

Finalmente, la totalidad de los encuestados afirmó que volvería a hospedarse en el Hotel Saratoga, dando como razones, la ubicación del hotel, el lujo y la tranquilidad que ofrece el mismo, así como la calidad humana y profesionalidad del personal.

\section{Debilidades, Amenazas, Fortalezas y Oportunidades del Hotel Saratoga en materia de Comunicación Promocional.}

Luego de haber realizado el diagnóstico del estado de la Comunicación Promocional en el Hotel Saratoga, los autores consideraron oportuno definir las principales debilidades, amenazas, fortalezas y oportunidades del hotel en lo que a esta materia se refiere, pues este análisis permitiría resumir de manera clara y certera, el estado real de la comunicación promocional. 
Debilidades

- Poco presupuesto destinado a la realización de acciones de promoción, debido a los escasos ingresos que reporta el hotel en estos momentos.

- La Comunicación Promocional no constituye una prioridad para el directivo, aunque es reconocida su importancia.

- Las acciones que se realizan en muchas ocasiones no se encuentran dirigidas hacia el público adecuado.

Amenazas

- La existencia de nuevos competidores en el área. Entre ellos la inauguración de los hoteles: Gran Manzana Kempinski y el Packard.

- Fuerte competencia que representa el sector privado (casas particulares) para la hotelería.

Fortalezas

- La preparación y profesionalidad que presenta el personal.

- El prestigio que se ganado el hotel desde su fundación.

- Excelente relación personal-directivo.

- El hotel hace uso de las tecnologías de la Información y la comunicación para promover sus productos-servicios.

Oportunidades

- La no inclusión del hotel en la lista negra de los EE.UU de los hoteles a no visitar en Cuba.

- El avance constante de las nuevas tecnologías de la información y la comunicación, que hace que todos los productos tengan que innovar constantemente.

\section{Conclusiones}

- La revisión bibliográfica y determinación de los fundamentos teóricometodológicos que sustentan la investigación, permitió sistematizar importantes conceptos estudiados, así como sentar las bases para el desarrollo de la investigación.

- La comunicación para cualquier entidad resulta de vital importancia ya que facilita en gran medida el funcionamiento de esta y la supervivencia de la organización a lo externo, pues consolida la Cultura Organizacional y el sentido de pertenencia de los trabajadores que la integran. En cuanto a la Comunicación promocional resulta necesaria para dar a conocer el producto-servicio que se quiere vender.

- El análisis de las encuestas y entrevistas realizadas en la instalación, permitió conocer las opiniones sobre la situación actual de la Comunicación Promocional 
en el hotel, así como determinar las debilidades y fortalezas que presenta el mismo, en aras de conocer en qué aspectos debe mejorar, para elevar no solo su imagen sino también las ventas del hotel.

- En el hotel Saratoga se le da gran importancia a la Comunicación Promocional y son empleadas por ello varias técnicas en aras de comercializar de una mejor forma sus productos, tratando de diferenciarse de la competencia

\section{Referencias Bibliográficas}

Ezquerra, G. y. (2007). Del Análisis a la Estrategia. Reposicionamiento del Destino Cuba en España. Tesis de Diploma. Facultad de Comunicación. Universidad de La Habana. La Habana.

Hotel-Saratoga.com. (s.f.). Obtenido de Política de privacidad: http:/www.hotelsaratoga.com

Ministerio del Turismo. (s.f.). lineamientos pertenecientes a la política de turismo.

Perelló, J. (2010). Comunicación Promocional en el Turismo. La Habana: Facultad de Turismo de la Universidad de La Habana.

Rivero, M. (2005). Marketing. Curso básico de Mercadotecnia. Instituto del Comercio exterior.

Sampieri, H. (2010). Metodología de la Investigación. Quinta Edición. Interamericanas Editores.

Saratoga, D. c. (s.f.). Manual de Identidad Corporativa del Hotel Saratoga.

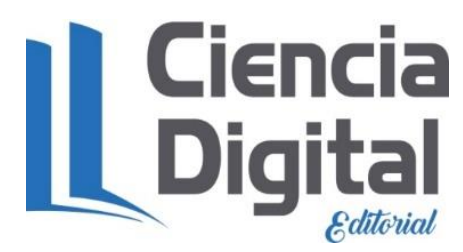




\section{PARA CITAR EL ARTÍCULO INDEXADO.}

Arceo Martínez, A., \& Malvarez Cuello, A. (2021). Diagnóstico del estado de la Comunicación Promocional del Hotel Saratoga. Explorador Digital, 5(2), 34-49. https://doi.org/10.33262/exploradordigital.v5i2.1651

\section{Liencia}

El artículo que se publica es de exclusiva responsabilidad de los autores y no necesariamente reflejan el pensamiento de la Revista Explorador Digital.

El artículo queda en propiedad de la revista y, por tanto, su publicación parcial y/o total en otro medio tiene que ser autorizado por el director de la Revista Explorador Digital.
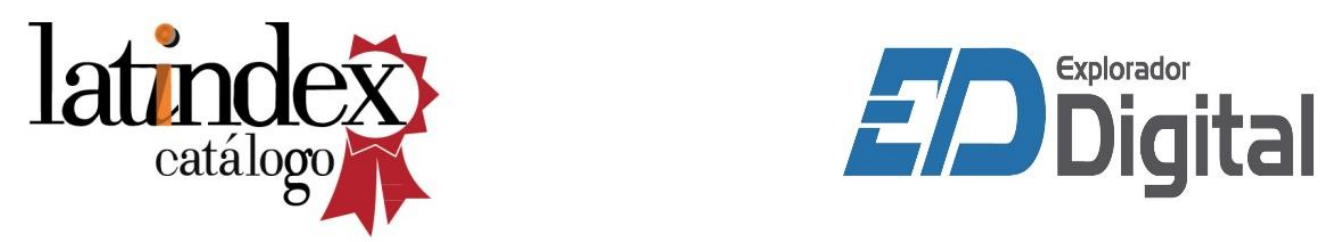